\title{
Combinatorial Therapy with Neurotrophins and cAMP Promotes Axonal Regeneration beyond Sites of Spinal Cord Injury
}

\author{
Paul Lu, ${ }^{1}$ Hong Yang, ${ }^{1}$ Leonard L. Jones, ${ }^{1,3}$ Marie T. Filbin, ${ }^{2}$ and Mark H. Tuszynski ${ }^{1,3}$ \\ ${ }^{1}$ Department of Neurosciences, University of California at San Diego, La Jolla, California 92093, ${ }^{2}$ Biology Department, Hunter College, New York, New York \\ 10021, and ${ }^{3}$ Veterans Affairs Medical Center, San Diego, California 92161
}

\begin{abstract}
Previous attempts to promote regeneration after spinal cord injury have succeeded in stimulating axonal growth into or around lesion sites but rarely beyond them. We tested whether a combinatorial approach of stimulating the neuronal cell body with cAMP and the injured axon with neurotrophins would propel axonal growth into and beyond sites of spinal cord injury. A preconditioning stimulus to sensory neuronal cell bodies was delivered by injecting cAMP into the L4 dorsal root ganglion, and a postinjury stimulus to the injured axon was administered by injecting neurotrophin-3 (NT-3) within and beyond a cervical spinal cord lesion site grafted with autologous bone marrow stromal cells. One to 3 months later, long-projecting dorsal-column sensory axons regenerated into and beyond the lesion. Regeneration beyond the lesion did not occur after treatment with cAMP or NT-3 alone. Thus, clear axonal regeneration beyond spinal cord injury sites can be achieved by combinatorial approaches that stimulate both the neuronal soma and the axon, representing a major advance in strategies to enhance spinal cord repair.
\end{abstract}

Key words: sensory systems; NT-3; dorsal columns; growth factors; cyclic nucleotides; axon

\section{Introduction}

The failure of axonal regeneration after CNS injury has been attributed to several factors, including (1) a lack of neurotrophic stimulation (Tuszynski et al., 1996; Liu et al., 1999), (2) an absence of permissive substrates to support axonal attachment and extension through the lesion site, (3) the presence of myelin(McKerracher et al., 1994; Schwab and Bartholdi, 1996; Filbin, 2003) and extracellular matrix-associated inhibitors (Fitch and Silver, 1999; Jones et al., 2002, 2003) in the injured region, and (4) a potential deficiency of intrinsic gene expression to support an active axonal growth state (Bomze et al., 2001). Targeting each of these mechanisms independently has resulted in increased axonal growth into or sprouting around spinal cord lesion sites (Schnell and Schwab, 1990; Tuszynski et al., 1996; Liu et al., 1999; Bradbury et al., 2002). However, the extent of this growth is generally either modest or fails to achieve substantial axonal growth beyond lesion sites.

The present study tested the hypothesis that combinatorial approaches to CNS regeneration, targeting several mechanisms that normally restrict axonal growth, would enhance axonal extension into and beyond spinal cord lesion sites. Recent studies

Received April 20, 2004; revised June 3, 2004; accepted June 4, 2004.

This work was supported by National Institutes of Health Grant R01 NS09881, the Veterans Administration, the Canadian Spinal Research Organization, and the Swiss Institute for Research into Paraplegia. We thank Lori Graham, Maya Culbertson, and Xiao He for outstanding technical assistance.

Correspondence should be addressed to Mark H. Tuszynski, Department of Neurosciences, University of California at San Diego, La Jolla, CA 92093-0626. E-mail: mtuszynski@ucsd.edu.

DOI:10.1523/JNEUROSCI.1492-04.2004

Copyright $\odot 2004$ Society for Neuroscience $\quad$ 0270-6474/04/246402-08\$15.00/0 have indicated that priming the sensory neuronal cell body either by a "preconditioning" lesion of the peripheral nerve (Neumann and Woolf, 1999) or by direct somal stimulation with cyclic nucleotides (Neumann et al., 2002; Qiu et al., 2002) can enhance central axonal sprouting after injury. The mechanism of this effect is likely to involve stimulation of endogenous cellular mechanisms that induce or support the axonal growth state and overcome local inhibition (Neumann et al., 2002; Qiu et al., 2002; Filbin, 2003). Separately, in vitro studies report that cyclic nucleotides (Song et al., 1998; Yuan et al., 2003) or neurotrophins (Gallo et al., 1997; Dickson, 2002; Dontchev and Letourneau, 2002) promote growth and turning of axon tips through rapid, local mechanisms. Indeed, injured adult axons extend along gradients of neurotrophic factors provided in an in vivo lesion site (Tuszynski et al., 1996; Liu et al., 1999; Lu et al., 2001; Ramer et al., 2002), but growth beyond the injury zone is generally not observed. The failure of axons to grow beyond an injury site after local growth factor delivery presumably is attributable to axonal entrapment within the high local concentrations of growth factors provided within the lesion zone. Provision of neurotrophic factor gradients beyond the injured region might be required to achieve axonal growth distal to the lesion site. Furthermore, it is possible that the combined stimulation of the neuronal soma together with the injured axon will be required to achieve a maximal growth state to propel distal axonal growth.

The present study tested this combinatorial hypothesis of regeneration by stimulating both the neuronal soma (using cAMP administration) and the injured axon (by local growth factor injections provided within and beyond cell grafts placed into cer- 
Table 1. Experimental groups

\begin{tabular}{lllll}
\hline Group & $\begin{array}{l}\text { DRG } \\
\text { injection }\end{array}$ & $\begin{array}{l}\text { Lesion site } \\
\text { injection }\end{array}$ & $\begin{array}{l}\text { Rostral } \\
\text { injection }\end{array}$ & $\begin{array}{l}\text { Number of } \\
\text { subjects }\end{array}$ \\
\hline CNN & CAMP & NT-3 plus MSC & NT-3 & 12 \\
CPP & CAMP & PBS plus MSC & PBS & 12 \\
PNN & PBS & NT-3 plus MSC & NT-3 & 12 \\
PNP & PBS & NT-3 plus MSC & PBS & 12 \\
PPP & PBS & PBS plus MSC & PBS & 12 \\
\hline
\end{tabular}

vical spinal cord lesion sites). Unlike provision of either cAMP alone or growth factors alone, significant axonal growth and bridging beyond the lesion site was achieved only using combinatorial strategies.

\section{Materials and Methods}

The dorsal-column sensory axon projection was used as a model system to test the hypothesis that combinatorial approaches to regeneration would propel axon growth beyond a lesion site. Previously, this system has exhibited sensitivity to cAMP administration (Neumann et al., 2002; Qiu et al., 2002) and to neurotrophin-3 (NT-3) (ElShamy and Ernfors, 1996; Liebl et al., 1997; Bradbury et al., 1999; Lu et al., 2003). Sixty adult female F344 rats were divided into five experimental groups (Table 1). All groups received injections of either dibutyryl-cAMP (50 $\mu \mathrm{g}$ in $2 \mu \mathrm{l}$ of solution) or vehicle (2 $\mu$ l of PBS) into bilateral L4 dorsal root ganglia (DRGs) to "precondition" the soma (Neumann et al., 2002; Qiu et al., 2002). Injections of this $50 \mu \mathrm{g} / 2 \mu \mathrm{l}$ dose of cAMP have previously been reported to neutralize the inhibitory effects of myelin on neurite outgrowth when administered in vivo to DRGs 1-7 d before explantation (Qiu et al., 2002). We injected cAMP into bilateral L4 DRGs $5 \mathrm{~d}$ before placing central spinal cord lesions at $\mathrm{C} 4$. This time point for cAMP pretreatment was chosen because it is well within the effective 1-7 $\mathrm{d}$ range over which cAMP has been shown to neutralize the inhibitory effects of myelin (Neumann et al., 2002; Qiu et al., 2002) and to mimic the preconditioning effects of peripheral nerve lesions on central axonal regeneration (Neumann and Woolf, 1999). Five days after cAMP administration, C4 dorsal columns were completely transected bilaterally using a Kopf wire knife device (David Kopf Instruments, Tujunga, CA) combined with tract external compression, as reported previously (Weidner et al., 1999; Jones et al., 2003) (see Fig. 1A). All groups then received implants of autologous bone marrow stromal cell (MSC) suspensions $\left(2 \times 10^{5}\right.$ cells in $2 \mu \mathrm{l}$ ) into the lesion site to establish a cellular matrix supportive of axon growth into the lesion (Hofstetter et al., 2002). In the absence of additional treatments, these MSCs support only modest host axon growth into the lesion site and not bridging beyond it (Hofstetter et al., 2002) (also see Results). MSCs were genetically modified to produce the reporter gene green fluorescent protein (GFP) to allow visualization. Animals also received injections of either NT-3 protein $(0.6 \mu \mathrm{g}$ in $2 \mu \mathrm{l})$ or PBS $(2 \mu \mathrm{l})$ in the lesion "cavity" containing the cell suspension. We hypothesized that immediate injection of NT-3 into the lesion cavity would promote dorsal-column sensory axon growth into the lesion site (Bradbury et al., 1999; Lu et al., 2003). Animals also received injections of either NT-3 $(1 \mu \mathrm{g}$ in $2 \mu \mathrm{l})$ or PBS $(2 \mu \mathrm{l})$ into the dorsal-column white matter of the host spinal cord $1.5 \mathrm{~mm}$ rostral to the lesion site 1 week after the 44 lesion (see Fig. 1A). A 1 week latency for NT-3 injection rostral to the lesion site was chosen to allow sufficient time for regenerating axons to extend through the graft-lesion site before exposure to potential chemotropic attraction by the rostral NT-3 injection. Animals were then functionally tested (see below), and after 1 or 3 months survival (Table 1 ), dorsal-column sensory axons were labeled transganglionically using cholera toxin-B (CTB) injections ( $2 \mu \mathrm{l}$ of $1 \%$ solution injected in each sciatic nerve) (Bradbury et al., 1999; Lu et al., 2003).

Marrow stromal cell culture. Primary adult female F344 MSCs were isolated as described by Azizi et al. (1998). To track grafted cells in vivo, passage 1 (P1) MSCs were transduced to express jellyfish GFP, as described previously (Lu et al., 2003). After expansion, P4 MSCs were grafted in vivo.
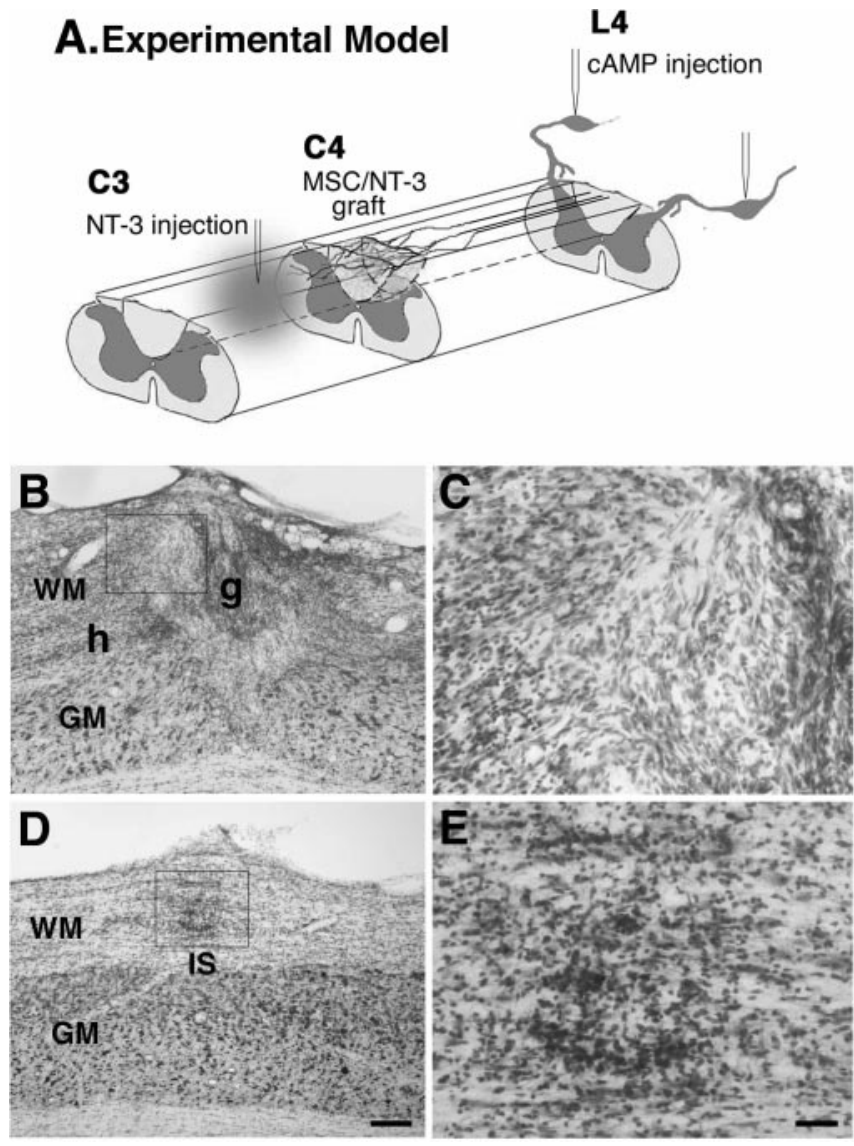

Figure 1. $A$, CAMP or PBS are injected into the $L 4$ DRG $5 \mathrm{~d}$ before transection of dorsalcolumn sensory axons at C4. NT-3 or PBS are injected into the lesion site, together with MSC, to provide a cellular matrix supporting axon penetration of the lesion site. One week later, NT-3 or PBS is injected $1.5 \mathrm{~mm}$ rostral to the lesion site to provide an attractive stimulus beyond the lesion in NT-3-injected subjects. $B-E$, Nissl sections show the morphology of the lesion site and graft $(B, C)$ and the rostral NT-3 injection site $(D, E)$. Boxed areas indicate regions of higher magnification. Good graft integration is evident, and minimal parenchymal disruption is seen at rostral injection site. g, MSC graft; $h$, host; WM, host white matter; GM, host gray matter; IS, rostral injection site. Scale bars: (in D) B, D, $200 \mu \mathrm{m}$; (in $E) C, E, 50 \mu \mathrm{m}$.

Immunolabeling. Light-level CTB immunolabeling was used to examine growth of dorsal-column sensory axons and to screen for spared axons in the medulla (polyclonal goat antibody, 1:10,000; List Biological Laboratories, Campbell, CA) (Lu et al., 2003). CTB-labeled sensory axons, GFP-expressing MSCs, and host astrocytes were detected by triple fluorescent labeling of every seventh section using antibodies against CTB (sensory axons, 1:5000), GFP (polyclonal antibody; 1:1500; Molecular Probes, Eugene, OR), and GFAP (monoclonal antibody; 1:750; Chemicon, Temecula, CA) incubated overnight at $4^{\circ} \mathrm{C}$. After washes, sections were incubated with cyanine 5-conjugated donkey anti-goat, fluorescein-conjugated donkey anti-rabbit, and Alexa 594-conjugated donkey anti-mouse secondary antibodies (1:200; Jackson ImmunoResearch, West Grove, PA) for $2.5 \mathrm{hr}$ at room temperature. Sections were coverslipped with Fluoromount G (Southern Biotechnology, Birmingham, AL).

Axon quantification. Sensory axon penetration into and beyond grafts was quantified in every subject in a series of three-in-seven CTB-labeled $30-\mu \mathrm{m}$-thick sagittal sections. Quantification was performed separately within grafts and in host spinal cord blocks located rostral to the lesion site at the following distances: $0-250,250-500,500-1000$, and $1000-$ $2000 \mu \mathrm{m}$. Within each block, the total number of CTB-labeled axons was counted in a microscopic field consisting of 100 boxes in a $10 \times 10$ matrix using $20 \times$ high-numerical aperture optics. Branched axons were counted only once. Data are presented as mean \pm SEM axon number. The host-lesion boundary was evident under phase-contrast optics as a 
distinct change in the appearance of neurons or white matter to the lesion cavity that contained uniform sheets of smaller, rounded cells. In addition, triple fluorescent labeling for CTBlabeled axons, GFP (MSCs), and GFAP (astrocytes) was used as a reference to confirm the location of the graft-host interface in each animal. Observers were blinded to group identity.

Evaluation of CTB transport to nucleus gracilis. Incomplete lesions would result in CTB labeling of the nucleus gracilis in the caudal medulla. In all subjects, the medulla was sectioned at $30 \mu \mathrm{m}$ intervals and every second section was labeled for CTB. Examiners blinded to group identity determined whether labeled axons or terminals were evident. Four intact animals underwent CTB injections of both sciatic nerves as positive controls for medullary labeling.

Functional analysis. The tape-removal test (Bradbury et al., 2002), horizontal ladder locomotion (Grill et al., 1997; Bradbury et al., 2002), and a rope-walking test (Kim et al., 2001) were used to assess functional performance in subjects surviving 3 months. A preoperative baseline was recorded followed by postoperative retesting once per week over the next 12 weeks (for details, see supplemental material, available at www.jneurosci.org/cgi/content/full/24/ 28/6402/DC1). Evaluators were blind to group identity.

Statistics. For axon density, multiple group comparisons were made by ANOVA with a significance criterion of $95 \%$. Post hoc differences were tested by Fisher's least square difference. Behavioral outcomes were assessed by repeatedmeasures ANOVA and post hoc Fisher's.

\section{Results}

Nissl staining showed excellent survival and integration of grafted autologous bone marrow stromal cells in the lesion site in all groups at time points of 1 and 3 months (Fig. 1B,C). The rostral site of NT-3 or vehicle injection showed a minimal gliotic reaction that did not differ in extent between NT-3- and vehicle-treated groups (Fig. $1 D, E$ ).

\section{Growth of sensory axons into the lesion site is augmented by either cAMP or NT-3}

The extent of dorsal-column sensory axon penetration into grafts placed in the lesion site differed as a function of experimental group (ANOVA; $p<0.0001)$ (Fig. 2) but not time $(p>0.05)$ (also see supplemental Figure 1, available at www.jneurosci.org/ cgi/content/full/24/28/6402/DC1). Thus, data for the 1 and 3 month time points were combined. Injections of cAMP into the DRG (group CPP) significantly elevated axon growth into the graft-lesion site compared with control-lesioned subjects that received PBS injections into DRGs and grafts (group PPP) ( $p=$ 0.02; post hoc Fisher's, group CPP compared with group PPP) (Fig. $2 B$ ). Local injection of NT-3 into the lesion site (group PNP) also significantly elevated sensory axon regeneration into the lesion site ( $p<0.001$; post hoc Fisher's compared with group PPP) (Fig. 2D) but did not significantly exceed the effect of cAMP injections into the DRG $(p>0.05$; post hoc Fisher's compared
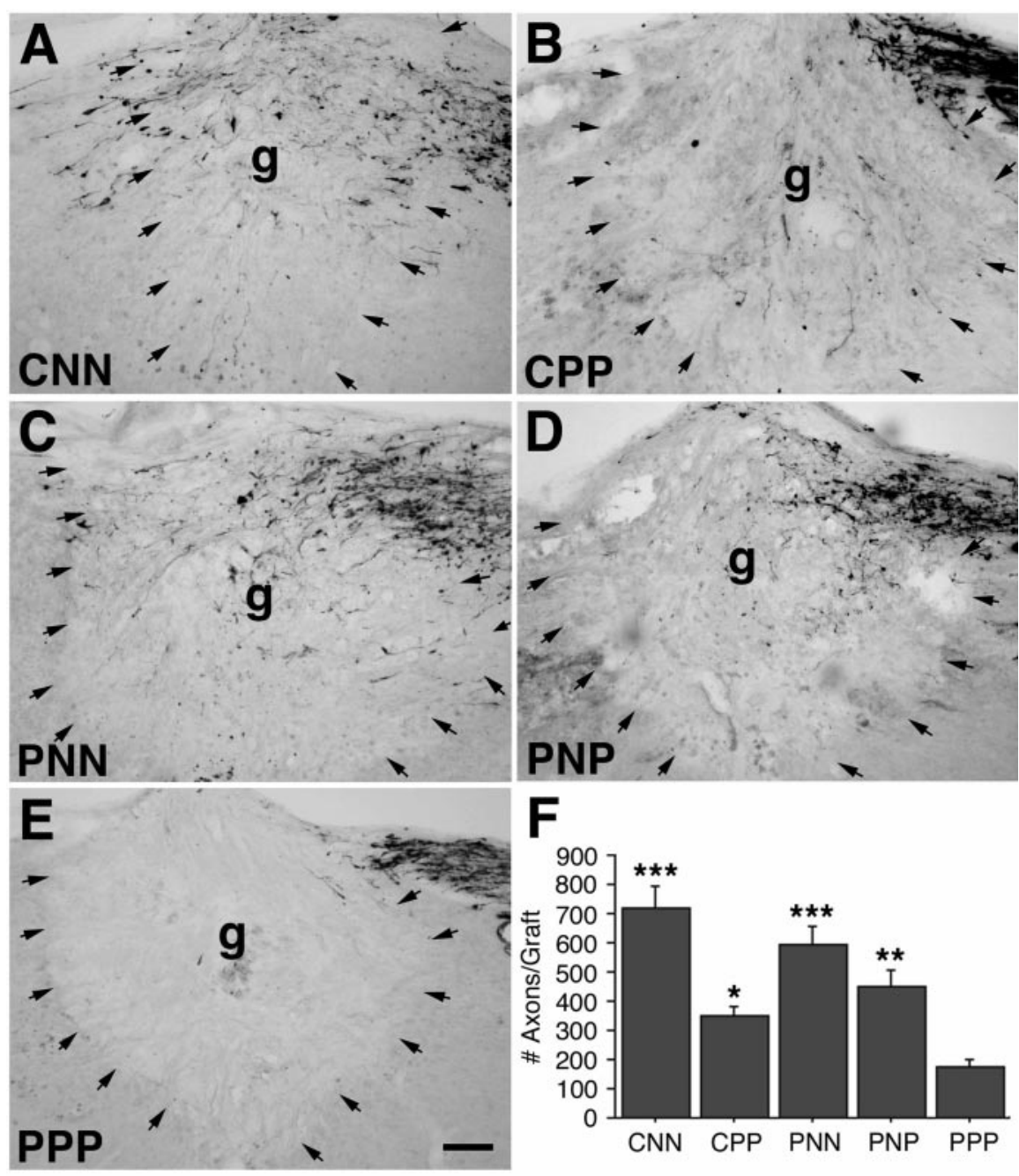

Figure 2. Sensory axon penetration of spinal cord lesion sites. A, CTB-labeled sensory axon penetration of MSC grafts in recipients of CAMP into DRGs plus NT-3 into and beyond the grafts. Sensory axon regeneration beyond the graft and into rostral host white matter is evident along the left side of the graft. In $A-E$, arrows indicate the host- graft interface. $\mathrm{g}$, Graft. Rostral is to Axons in this group modestly reenter rostral host tissue (Fig. 3B).D, Sensory axons primarily penetrate the caudal aspect of the MSC (he graft only, without CAMP injection into the DRG. E, Sensory axons rarely axons in the lesion site indicates that CAMP or neurotrophins augment axon penetration of the graft (overall ANOVA; $p<0.0001$ ). Asterisks indicate differences between indicated groups and control PPP. ${ }^{*} p<0.05 ;{ }^{* *} p<0.001 ;{ }^{* * *} p<0.0001$.

with group $\mathrm{CPP}$ ). Injection of NT-3 into and beyond the lesion site (groups PNN and CNN) further increased sensory axon growth into the lesion site ( $p<0.0001$ relative to group PPP) (Fig. $2 A, C$ ). The combined delivery of cAMP and NT-3 (CNN) did not exceed the effect of NT-3 alone (PNN) in promoting axonal growth into the lesion site ( $p>0.05$; post hoc Fisher's). Thus, stimulation of either the sensory soma with cAMP or the injured axon with NT-3 promoted axonal growth into the permissive milieu of the marrow stromal cell graft in the lesion site.

\section{Combination therapy with cAMP plus NT-3 gradients} promotes significant axonal growth beyond the lesion site Axonal egress from the graft-lesion site was observed to a consistent and significant extent only in subjects that received combination therapy with cAMP stimulation to the DRG soma and NT-3 injections within and beyond the C4 cervical lesion site (CNN group) (Figs. 3-7). The number of CTB-labeled axons was 


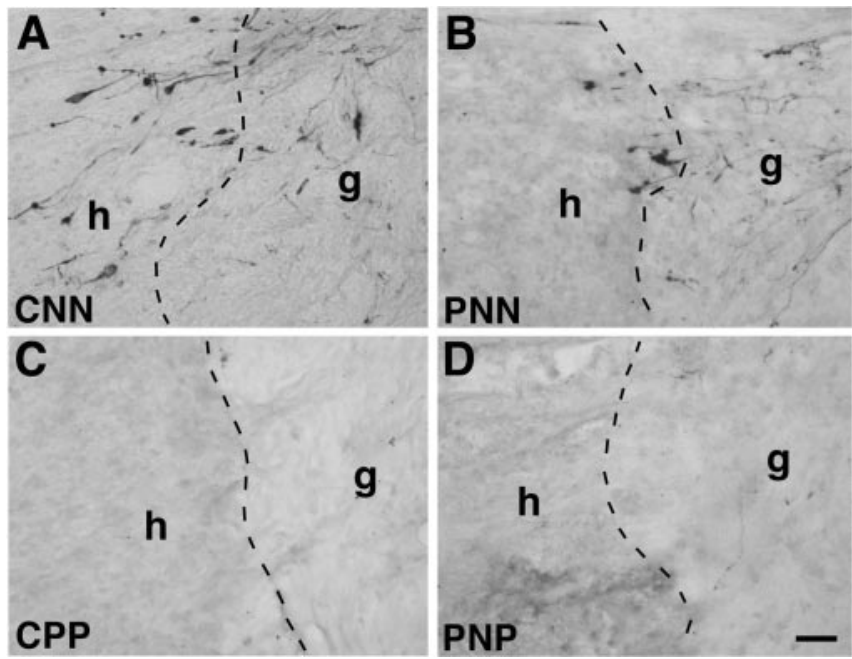

Figure 3. Emergence of CTB-labeled sensory axons beyond the rostral MSC graft-host interface. $A$, CTB-labeled sensory axons regenerate beyond the graft-host interface and enter rostral host white matter after combined administration of intraganglionic CAMP and axonal application of NT-3. The dashed line indicates the graft-host interface. $g$, Graft; $h$, host. $B$, Sensory axons extend for short distances beyond the rostral graft-host interface in subjects that receive axonal application of NT-3 within and beyond the lesion site, without CAMP injection into the DRG. However, the extent of growth in this group is modest. C, D, Sensory axons fail to regenerate in other groups, including recipients of cAMP injections alone into the DRG (C), recipients of NT-3 alone into the lesion site $(D)$, or recipients that receive neither CAMP nor NT-3 (data not shown). Scale bar: (D) $A-D, 35 \mu \mathrm{m}$.

measured at intervals of 250, 500, 1000, and $2000 \mu \mathrm{m}$ beyond the lesion. Axons clearly emerged from the lesion site in only two groups of subjects: animals that received injections of cAMP plus NT-3 gradients beyond the lesion site $(\mathrm{CNN})$ and animals that received NT-3 injections into and beyond the $\mathrm{C} 4$ lesion site with PBS injections into the DRG (PNN) (Figs. 3-7). However, the number of axons was far greater in the CNN group $(p<0.005$; post hoc Fisher's comparing $\mathrm{CNN}$ with $\mathrm{PNN}$ at each distance) (Figs. 3-5), and every animal in the CNN group exhibited growth beyond the lesion site. Axonal bridging was not observed in any group lacking NT-3 injections beyond the lesion site (PNP, CPP, PPP) (Figs. 3, 5). Thus, the combination of cAMP plus NT-3 injections into and beyond the lesion site resulted in the greatest degree of axonal regeneration.

The maximal distance of axonal regeneration beyond the lesion zone in recipients of combined treatment with cAMP and NT-3 was 1.5-2 mm, corresponding to the location of the rostral NT-3 injection (Figs. 4-6). Growth beyond the rostral NT-3 injection site was not observed. In contrast, the only other group to exhibit axonal growth beyond the lesion zone, PNN, showed virtually no axon growth beyond a distance of $0.5 \mathrm{~mm}$ rostral to the lesion (Figs. 3, 5). Of note, axonal growth beyond the lesion zone in the CNN and PNN groups occurred through host white matter of the dorsal columns, the prelesion position of this projecting tract (Figs. 3-7). The morphology of sensory axons reaching the rostral NT-3 injection site consisted of thick as well as fine and varicose axons (Figs. 4, 6A). Also evident only at the rostral NT-3 injection site were CTB-labeled sensory axons with highly branched morphology and aggregates of neuritic labeling, suggesting potential termination of growth (Fig. $6 B-D$ ). As noted below, all CTB-labeled axons beyond the lesion site showed irregular profiles with frequent turns, unlike preinjured axons that exhibit a strictly linear morphology.

Confocal triple labeling for CTB-labeled sensory axons, grafted marrow stromal cells (GFP fluorescence), and GFAP confirmed the presence of axons extending rostral to the lesion site and beyond implants of GFP-labeled marrow stromal cells (Fig. 7). Sensory axons extended through the GFAP-rich "glial scar" (Fig. $7 B$ ). MSCs remained within the lesion site, rarely migrating only short distances $(<150 \mu \mathrm{m})$ from the lesion boundary (Fig. $7 A, B)$.

\section{Evidence for completeness of dorsal-column lesions}

Three observations suggested that axons actually regenerated through the lesion site and were not merely spared as a result of incomplete lesions. First, the medulla was sectioned microscopically to determine whether any terminating axons were present in the nucleus gracilis (reflecting potentially spared axons) (Fig. 8 ). No labeled axons in the nucleus gracilis were observed in any lesioned subject, whereas dense labeling was readily detected in four control, intact animals. Thus, axons extending beyond the lesion site were not spared, nor did regenerating axons in the cAMP plus NT-3 group extend completely to their natural target in the caudal medulla. Second, CTB-labeled axons were observed to directly emerge across the lesion-host interface at many different dorsoventral levels of the graft (Figs. 3, 4, 7), including mid-portions of the graft that were distant from either the top or the bottom of the lesion cavity. Hence, axons emerged from the "core" of the graft and not only from the most dorsal or ventral aspects of the lesion site where spared axons would most likely be located, if present. Third, regenerating axons often exhibited a rather varicose morphology, and their course of growth was not strictly linear, unlike spared axons that typically exhibit a more smooth and linear orientation.

\section{Functional outcomes}

Significant functional differences among lesioned groups were not observed on the tape-removal task, horizontal ladder, or rope task (Grill et al., 1997; Kim et al., 2001; Bradbury et al., 2002) from 1 week to 3 months postoperatively (see supplemental Figure 2, available at www.jneurosci.org/cgi/content/ full/24/28/6402/DC1).

\section{Discussion}

These findings indicate that combinatorial approaches to spinal cord regeneration (Schwab and Bartholdi, 1996; Geller and Fawcett, 2002) can promote axonal bridging beyond sites of spinal cord injury (SCI) and through host white matter. cAMP stimulation of the neuronal soma has been shown previously to neutralize the inhibitory effect of myelin-associated inhibitors such as Nogo and myelin-associated glycoprotein on axon growth in vitro (Neumann et al., 2002; Qiu et al., 2002). cAMP stimulation of the cell body in the absence of neurotrophin treatment, although sufficient to promote sensory axonal sprouting beyond a spinal cord injury site through spared host gray matter (Neumann et al., 2002; Qiu et al., 2002), was not sufficient in this study to promote axonal bridging through an autologous cell graft placed within the lesion cavity. Because gray matter is rarely spared at human spinal cord injury sites, cAMP therapy alone might not be practical in many human injuries, and some type of bridging substrate will likely be required to support host axonal regeneration. Like cAMP administration, neurotrophin stimulation of the axon without concomitant intraganglionic cAMP was not capable of promoting substantial axon growth beyond the cell graft in the lesion cavity. Furthermore, the injection of NT-3 above the lesion site was required to support significant growth beyond the lesion. The combination of somal stimulation by 



Figure 4. Sensory axons regenerate beyond spinal cord lesion sites after combined administration of intraganglionic cAMP and axonal application of NT-3. A, Lower-magnification view of a sagittal section of spinal cord illustrating (TB-labeled dorsal-column sensory axons approaching the lesion site (right, arrowhead), the MSC graft in the lesion cavity ( $g$; arrows indicate the host- graft interface), and the region rostral to the lesion site (left). IS, Rostral injection site of NT-3 (also indicated by the large arrow); $g$, graft; h, host. B, Boxed area of graft. CTB-labeled sensory axons penetrate the graft in the lesion site. C, Higher magnification of box 1 from $A$, demonstrating crossing of CTB-labeled axons from the graft into the host white matter beyond the graft. The dashed line indicates the host-graft interface. This crossing occurs at a point well away from the dorsal or ventral lesion regions in which spared axons might be present. D, Higher magnification of box 2 from $A$, demonstrating several varicose (TB-labeled axons extending $0.5-0.7 \mathrm{~mm}$ beyond the lesion site (arrowheads). E, Higher magnification of box 3 from $A$, showing additional axons extending under the dorsal aspect of the spinal cord beyond the lesion site (arrowheads). Scale bars: $A, 280 \mu \mathrm{m}$; (in $E) B-E, 44 \mu \mathrm{m}$. 
A. Axons beyond graft (250um)

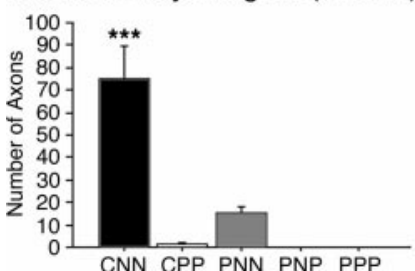

B. Axons beyond graft (500um)

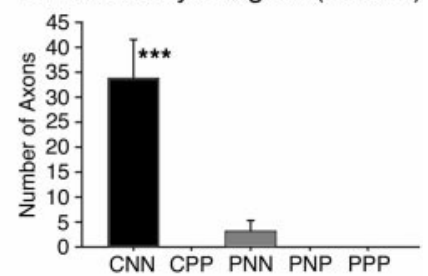

C.Axons beyond graft (1mm)



D. Axons beyond graft $(2 \mathrm{~mm})$

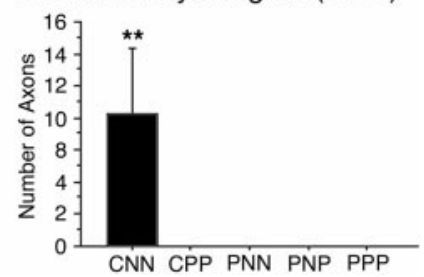

Figure 5. Quantification of sensory axon regeneration beyond the MSC graft. Axons extend beyond the graft only in subjects that contain NT-3 gradients distal to the lesion site. The quantity and distance of this growth is significantly amplified after combined somal stimulation with cAMP and axonal stimulation with NT-3. ${ }^{* *} p<0.005$ from 1000 to $2000 \mu \mathrm{m}$ distal to the graft; ${ }^{* * *} p<0.0001$ at 250,500 , and $1000 \mu \mathrm{m}$.

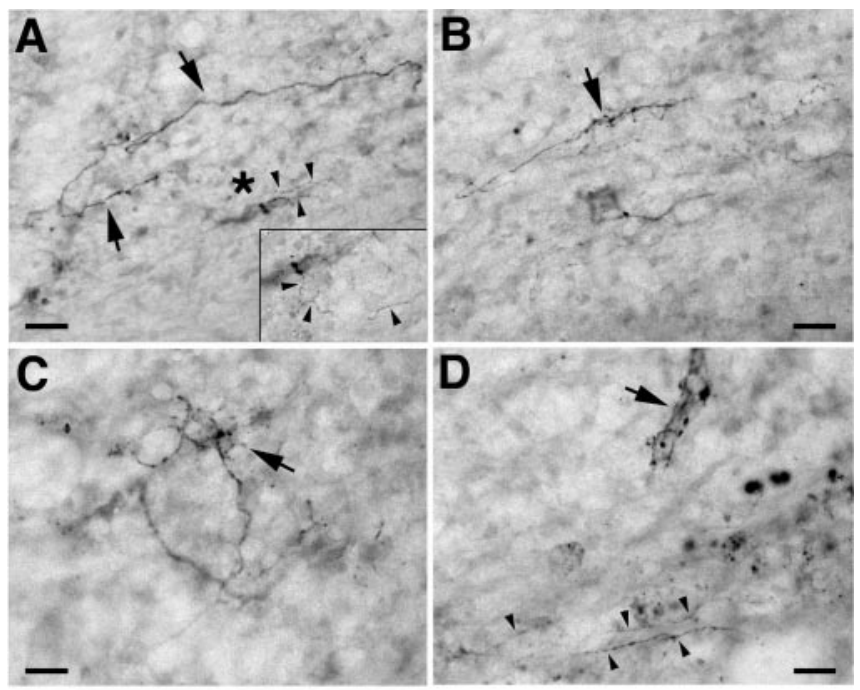

Figure 6. Morphology of regenerating sensory axons adjacent to the rostral NT-3 injection site in recipients of CAMP and NT-3 injections. A, Large-diameter (arrows) and small-diameter (arrowheads) CTB-labeled sensory axons are evident with nonlinear morphology, branching, and several varicosities. The axon indicated by an asterisk is shown at higher magnification and a slightly different focal plane in the inset, where additional fine neuritic processes are evident. $B, C$, Regenerated axons exhibit numerous varicosities, short branches, and aggregates of small neurites (arrow). D, Another example of numerous small varicosities (arrow) and branched axons (arrowheads) adjacent to a rostral NT-3 injection site (IS). Scale bars: $A, B, 15 \mu \mathrm{m} ; C, 10$ $\mu \mathrm{m} ; D, 13 \mu \mathrm{m}$.

cAMP and axonal stimulation with a neurotrophin gradient clearly promoted the greatest degree of axonal bridging in the spinal cord beyond a lesion site containing an autologous cell graft.

Of note, it does not appear likely that axonal growth beyond the lesion site in the cAMP plus NT-3 group was simply a function of a greater number of axons filling the graft in the lesion site, presenting a greater potential pool of axons to regenerate beyond the lesion site. The mean density of axons within lesion site grafts differed by only $18 \%$ in animals that received cAMP in the DRG plus NT-3 in the graft and beyond (CNN; $719 \pm 77$ axons in graft) compared with animals that received PBS in the DRG plus NT-3 in the graft and beyond (PNN; $592 \pm 62$ axons in graft). However, axon growth beyond the lesion site was augmented at least fivefold at every spinal level rostral to the lesion site in recipients of cAMP plus NT-3 (CNN group) compared with the PBS plus NT-3 (PNN) group (Fig. 5). This finding suggests that cAMP administration had a proportionately greater effect on the augmentation of axonal regeneration through the normally inhibitory milieu of host white matter than through the environment of the implanted marrow cells in the lesion site, a finding consistent with in vitro observations (Qiu et al., 2002) and in vivo sprouting studies (Neumann et al., 2002; Qiu et al., 2002).

The observed degree of axonal regeneration in this study was not sufficient to support functional recovery, likely because regenerating sensory axons did not reach their gracile nucleus target. Achieving growth to the target would require extended gradients of growth factors, a potentially attainable objective using methods such as in vivo growth factor gene delivery. Notably, even short-distance motor axonal growth beyond a lesion site could be sufficient to promote functional recovery on the basis of reinnervation of motor units located just distal to a lesion site.

Although this study suggests that stimulation of both the neuronal soma and axon primes the cell to an optimal growth state, this dual stimulation might potentially be generated with means other than CAMP and neurotrophin injections. For example, injections of a neurotrophin at both sites or cAMP-stimulating compounds at both sites may be sufficient. Alternatively, a greater magnitude of stimulation or greater duration of stimulation at either site alone might also support distal bridging. These possibilities will be fruitful subjects of additional study.

Although previous studies have reported axonal bridging beyond sites of spinal cord injury, the present findings are unique from four perspectives. First, we observed axonal regeneration beyond the lesion site and through the inhibitory milieu of adult white matter. To our knowledge, no previous report has demonstrated axonal regeneration through cell grafts placed in lesion sites and continued growth beyond the lesion through host white matter. It has been reported previously that neutralizing antibodies or peptides directed against the myelin-associated inhibitor Nogo or its receptors can promote axonal sprouting through spared host gray matter (but not through grafts) at a lesion site, and that axonal regeneration will continue caudally through white matter (Schnell and Schwab, 1990; Brosamle et al., 2000). However, two recent reports raise the possibility that inactivation of the Nogo gene alone, a primary white-matter inhibitor, may be insufficient to support extensive white-matter axonal regeneration (Simonen et al., 2003; Woolf, 2003; Zheng et al., 2003). A third report of Nogo inactivation demonstrated that regeneration was enhanced in postnatal but not adult mice (Kim et al., 2003). The present approach presents an alternative means of targeting white-matter inhibition by modifying intrinsic neuronal responses to inhibitors (Neumann et al., 2002; Qiu et al., 2002) and by balancing local inhibition (Jones et al., 2003) with growth factors provided at the tips of injured axons. This may therefore provide a means of more broadly targeting an array of inhibitory molecules present at sites of CNS injury.

Second, the observed growth of host axons beyond the lesion site in this study occurred after complete lesions of bilateral projections of the injured pathway, with little chance that the lesions were incomplete. Lesion completeness was verified by the total absence of CTB labeling in the nucleus gracilis of every lesioned subject in this study. Furthermore, the morphology of the ex- 
tending axons (nonlinear, varicose) was inconsistent with spared axons. The few preceding studies that have raised the possibility of axonal regeneration beyond implants in spinal cord lesion sites have used incomplete lesions of the systems under study or shown axonal growth at the ventral or dorsal aspect of the lesion cavity, raising the possibility of axonal sparing rather than regeneration. In contrast, regenerating axons in this study emerged from the core, midportion of the graft (Figs. 3, 4, 7), where the chance of artifactual sparing is extremely remote. Hence, we show unequivocal axon growth beyond a cellular bridge in a lesion site, a major advance for the field of spinal cord repair.

Third, several dozen axons from a longprojecting tract extended beyond the lesion after injections of cAMP into just one dorsal root ganglion level, $\mathrm{L} 4$. The number of responding axons appears to substantially exceed previous reports of potential axonal bridging beyond regions of cell implants in SCI lesion sites, which generally have shown individual axons (RamonCueto et al., 2000) or have traced local axons rather than long-projecting systems (Bamber et al., 2001). It is anticipated that greater numbers of extending axons would be observed if multiple DRG levels were targeted.

Fourth, to our knowledge this is the first report that a strategy of augmenting the intrinsic growth state of the neuron by somal stimulation can be combined with stimulation to the tip of the injured axon to substantially augment plasticity and regeneration after CNS injury. Combinatorial approaches to promote axonal regeneration in the CNS have been put forth previously as essential in addressing the multiple problems of reconstituting a matrix at the lesion site that can support axonal extension, providing a growth stimulus to the axon and neutralizing inhibition to support growth into and beyond the lesion site. The present paradigm may represent the first such combinatorial approach to provide proof of principle for these objectives in the spinal cord. A recent study in an optic nerve crush model also reported that combinatorial therapy, namely Nogo neutralization and provision of a growth stimulus to retinal ganglion cells, supported regrowth of injured axons (Fischer et al., 2004). In agreement with the present findings, Pearse et al. (2004) recently reported that combinatorial treatment with Schwann cell grafts and elevations in cAMP levels reduced lesion size and improved functional outcomes after contusive SCI. Collectively, this scenario defines potential approaches to clinical translation, as continuing studies define the precise means and requirements for timing of cAMP stimulation and growth factor delivery to injury sites.

\section{References}

Azizi SA, Stokes D, Augelli BJ, DiGirolamo C, Prockop DJ (1998) Engraftment and migration of human bone marrow stromal cells implanted in the brains of albino rats-similarities to astrocyte grafts. Proc Natl Acad Sci USA 95:3908-3913.

Bamber NI, Li H, Lu X, Oudega M, Aebischer P, Xu XM (2001) Neurotrophins BDNF and NT-3 promote axonal re-entry into the distal host spinal
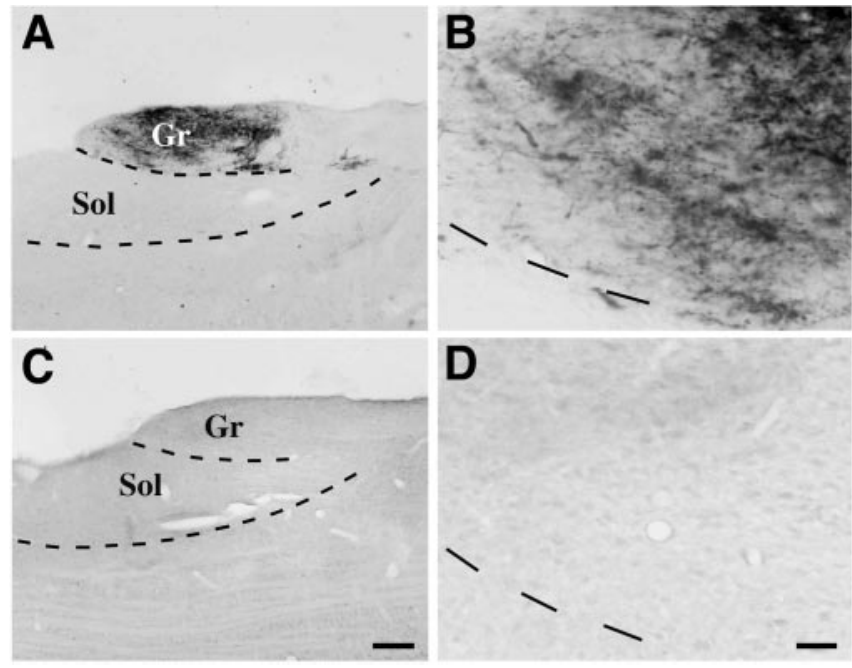

Figure 8. Sensory axon labeling in the nucleus gracilis. $A, B$, In unlesioned, intact animals, dense terminals of CTB-labeled axons are visible in sagittal sections of the caudal medulla within the nucleus gracilis (Gr) (above the top dashed lines). Sol, Solitary nucleus (between the top and bottom dashed lines). C, D, CTB-labeled axon terminals were not present in any lesioned animal, indicating both that lesions were complete and that regenerating axons did not resynapse within their normal targets. Scale bars: (in C) A, C, $177 \mu \mathrm{m}$; (in D) B, D, $27.5 \mu \mathrm{m}$.

cord through Schwann cell-seeded mini-channels. Eur J Neurosci 13:257-268.

Bomze HM, Bulsara KR, Iskandar BJ, Caroni P, Skene JH (2001) Spinal axon regeneration evoked by replacing two growth cone proteins in adult neurons. Nat Neurosci 4:38-43.

Bradbury EJ, Khemani S, King VR, Priestley JV, McMahon SB (1999) NT-3 
promotes growth of lesioned adult rat sensory axons ascending in the dorsal columns of the spinal cord. Eur J Neurosci 11:3873-3883.

Bradbury EJ, Moon LD, Popat RJ, King VR, Bennett GS, Patel PN, Fawcett JW, McMahon SB (2002) Chondroitinase ABC promotes functional recovery after spinal cord injury. Nature 416:636-640.

Brosamle C, Huber AB, Fiedler M, Skerra A, Schwab ME (2000) Regeneration of lesioned corticospinal tract fibers in the adult rat induced by a recombinant, humanized IN-1 antibody fragment. J Neurosci 20:8061-8068.

Dickson BJ (2002) Molecular mechanisms of axon guidance. Science 298:1959-1964.

Dontchev VD, Letourneau PC (2002) Nerve growth factor and semaphorin $3 \mathrm{~A}$ signaling pathways interact in regulating sensory neuronal growth cone motility. J Neurosci 22:6659-6669.

ElShamy WM, Ernfors PA (1996) Local action of neurotrophin-3 prevents the death of proliferating sensory neuron precursor cells. Neuron 16:963-972.

Filbin MT (2003) Myelin-associated inhibitors of axonal regeneration in the adult mammalian CNS. Nat Rev Neurosci 4:703-713.

Fischer D, He Z, Benowitz LI (2004) Counteracting the Nogo receptor enhances optic nerve regeneration if retinal ganglion cells are in an active growth state. J Neurosci 24:1646-1651.

Fitch MT, Silver J (1999) Beyond the glial scar. In: CNS regeneration: basic science and clinical aspects (Tuszynski MH, Kordower JA, eds), pp 55-81. San Diego: Academic.

Gallo G, Lefcort FB, Letourneau PC (1997) The trkA receptor mediates growth cone turning toward a localized source of nerve growth factor. J Neurosci 17:5445-5454.

Geller HM, Fawcett JW (2002) Building a bridge: engineering spinal cord repair. Exp Neurol 174:125-136.

Grill R, Murai K, Blesch A, Gage FH, Tuszynski MH (1997) Cellular delivery of neurotrophin-3 promotes corticospinal axonal growth and partial functional recovery after spinal cord injury. J Neurosci 17:5560-5572.

Hofstetter CP, Schwarz EJ, Hess D, Widenfalk J, El Manira A, Prockop DJ, Olson L (2002) Marrow stromal cells form guiding strands in the injured spinal cord and promote recovery. Proc Natl Acad Sci USA 99:2199-2204.

Jones LL, Yamaguchi Y, Stallcup WB, Tuszynski MH (2002) NG2 is a major chondroitin sulfate proteoglycan produced after spinal cord injury and is expressed by macrophages and oligodendrocyte progenitors. J Neurosci 22:2792-2803.

Jones LL, Sajed D, Tuszynski MH (2003) Axonal regeneration through regions of chondroitin sulfate proteoglycan deposition after spinal cord injury: a balance of permissiveness and inhibition. J Neurosci 23:9276-9288.

Kim D, Schallert T, Liu Y, Browarak T, Nayeri N, Tessler A, Fischer MM (2001) Transplantation of genetically modified fibroblasts expressing BDNF in adult rats with a subtotal hemisection improves specific motor and sensory functions. Neurorehabil Neural Repair 15:141-150.

Kim JE, Li S, GrandPre T, Qiu D, Strittmatter SM (2003) Axon regeneration in young adult mice lacking Nogo-A/B. Neuron 38:187-199.

Liebl DJ, Tessarollo L, Palko ME, Parada LF (1997) Absence of sensory neurons before target innervation in brain-derived neurotrophic factor-, neurotrophin 3-, and TrkC-deficient embryonic mice. J Neurosci 17:9113-9121.

Liu Y, Kim D, Himes BT, Chow SY, Schallert T, Murray M, Tessler A, Fischer I (1999) Transplants of fibroblasts genetically modified to express BDNF promote regeneration of adult rat rubrospinal axons and recovery of forelimb function. J Neurosci 19:4370-4387.
Lu P, Blesch A, Tuszynski M (2001) Neurotrophism without neurotropism: BDNF promotes survival but not growth of lesioned corticospinal neurons. J Comp Neurol 436:456-470.

Lu P, Jones J, Snyder E, Tuszynski M (2003) Neural stem cells constitutively secrete neurotrophic factors and promote robust host axonal growth after spinal cord injury. Exp Neurol 181:115-129.

McKerracher L, David S, Jackson DL, Kottis V, Dunn RJ, Braun PE (1994) Identification of myelin-associated glycoprotein as a major myelinderived inhibitor of neurite growth. Neuron 13:805-811.

Neumann S, WoolfCJ (1999) Regeneration of dorsal column fibers into and beyond the lesion site following adult spinal cord injury. Neuron 23:83-91.

Neumann S, Bradke F, Tessier-Lavigne M, Basbaum AI (2002) Regeneration of sensory axons within the injured spinal cord induced by intraganglionic cAMP elevation. Neuron 34:885-893.

Pearse DD, Pereira FC, Marcillo AE, Bates ML, Berrocal YA, Filbin MT, Bunge MB (2004) cAMP and Schwann cells promote axonal growth and functional recovery after spinal cord injury. Nat Med 10:610-616.

Qiu J, Cai D, Dai H, McAtee M, Hoffman PN, Bregman BS, Filbin MT (2002) Spinal axon regeneration induced by elevation of cyclic AMP. Neuron 34:895-903.

Ramer MS, Bishop T, Dockery P, Mobarak MS, O’Leary D, Fraher JP, Priestley JV, McMahon SB (2002) Neurotrophin-3-mediated regeneration and recovery of proprioception following dorsal rhizotomy. Mol Cell Neurosci 19:239-249.

Ramon-Cueto A, Cordero MI, Santos-Benito FF, Avila J (2000) Functional recovery of paraplegic rats and motor axon regeneration in their spinal cords by olfactory ensheathing glia. Neuron 25:425-435.

Schnell L, Schwab ME (1990) Axonal regeneration in the rat spinal cord produced by an antibody against myelin-associated neurite growth inhibitors. Nature 343:269-272.

Schwab ME, Bartholdi D (1996) Degeneration and regeneration of axons in the lesioned spinal cord. Physiol Rev 76:319-370.

Simonen M, Pedersen V, Weinmann O, Schnell L, Buss A, Ledermann B, Christ F, Sansig G, van der Putten H, Schwab ME (2003) Systemic deletion of the myelin-associated outgrowth inhibitor Nogo-A improves regenerative and plastic responses after spinal cord injury. Neuron 38:201-211.

Song H, Ming G, He Z, Lehmann M, McKerracher L, Tessier-Lavigne M, Poo M (1998) Conversion of neuronal growth cone responses from repulsion to attraction by cyclic nucleotides. Science 281:1515-1518.

Tuszynski MH, Gabriel K, Gage FH, Suhr S, Meyer S, Rosetti A (1996) Nerve growth factor delivery by gene transfer induces differential outgrowth of sensory, motor, and noradrenergic neurites after adult spinal cord injury. Exp Neurol 137:157-173.

Weidner N, Grill RJ, Tuszynski MH (1999) Elimination of basal lamina and the collagen "scar" after spinal cord injury fails to augment corticospinal tract regeneration. Exp Neurol 160:40-50.

Woolf CJ (2003) No Nogo: now where to go? Neuron 38:153-156.

Yuan XB, Jin M, Xu X, Song YQ, Wu CP, Poo MM, Duan S (2003) Signalling and crosstalk of Rho GTPases in mediating axon guidance. Nat Cell Biol $5: 38-45$.

Zheng B, Ho C, Li S, Keirstead H, Steward O, Tessier-Lavigne M (2003) Lack of enhanced spinal regeneration in Nogo-deficient mice. Neuron $38: 213-224$. 